\title{
LAS RELACIONES PARLAMENTO-GOBIERNO DURANTE EL ESTADO DE ALARMA POR COVID-19
}

\author{
Andrés Iván Dueñas Castrillo \\ Profesor de Derecho Constitucional \\ Universidad de Valladolid
}

\begin{abstract}
Cómo citar este artículo / Citation: Dueñas Castrillo, A.I. (2020). Las relaciones ParlamentoGobierno durante el estado de alarma por covid-19. Biglino Campos, P.; Durán Alba, F. Los Efectos Horizontales de la COVID sobre el sistema constitucional, Colección Obras colectivas, Fundación Manuel Giménez Abad, Zaragoza. DOI: https://doi.org/10.47919/FMGA.OC20.0004
\end{abstract}

SUMARIO: I. INTRODUCCIÓN. II. BREVES APUNTES GENERALES SOBRE LA FUNCIÓN DE CONTROL. III. REACCIÓN DE LOS PARLAMENTOS ANTE LA COVID-19. ESPECIAL REFERENCIA AL CONGRESO DE LOS DIPUTADOS. IV. LA FISCALIZACIÓN AL GOBIERNO DURANTE EL ESTADO DE ALARMA POR COVID-19. V. CONCLUSIONES. VI BIBLIOGRAFÍA.

\section{INTRODUCCIÓN}

Durante el estado de alarma por covid-19 las relaciones entre el Parlamento y el Gobierno han variado muy sensiblemente en comparación con una situación de normalidad. Hoy en día se puede llegar a afirmar que el Ejecutivo tiene mayor capacidad para legislar que el Legislativo, por lo que la función de control se ha convertido en la más importante de las tareas con las que cuentan las Asambleas Legislativas.

En una situación donde el Gobierno ha contado con un poder mucho mayor, su fiscalización debería haber sido sumamente relevante desde el inicio del estado de alarma, más aún si se tiene en cuenta el papel que otorga el artículo 116 CE al Congreso de los Diputados en cualquiera de los estados que contempla dicho precepto. Sin embargo, debido al contexto generado por la pandemia, el Parlamento casi desapareció en los primeros días de estado de alarma, aunque 
fundación

Manuel Giménez Abad

deEstudios Parlamentariosydel Estado Autonómico

con el paso de las semanas y las sucesivas prórrogas de tal estado se fue volviendo a una situación donde la fiscalización al Ejecutivo pasó a ser más significativa.

Nuestros Parlamentos, tanto en el ámbito autonómico como en el estatal, han dado respuestas diferentes para afrontar el período de confinamiento que se decretó durante el estado de alarma. En este capítulo se pretende hacer un pequeño balance de las diversas soluciones que se han planteado y hacer un análisis más concreto de la fiscalización al Gobierno estatal por las Cortes Generales durante el estado de alarma por covid-19, no sin antes hacer una pequeña mención a la actualidad de la función de control de las Cámaras.

\section{BREVES APUNTES GENERALES SOBRE LA FUNCIÓN DE CONTROL}

La noción clásica de los liberales sobre el Parlamento ha cambiado radicalmente. Antes se entendía como una reunión de representantes libres e iguales que encarnaban la soberanía de la nación y que debían controlar a un Gobierno del que desconfiaban para equilibrar su posición respecto al Ejecutivo (Fernández Sarasola, 2000: 91). Pero ahora "los verdaderos destinatarios de la discusión son los ciudadanos y no tanto unos parlamentarios a los que hay que persuadir o con los que hay que colaborar para tomar la decisión más racional" (Requejo Rodríguez, 2016: 107).

Uno de los principales problemas en las relaciones entre Gobierno y Parlamento en la actualidad es que el Ejecutivo controla al Legislativo a través de una mayoría parlamentaria que en muchas ocasiones actúa como rodillo ante la minoría. Es cierto que la actual fragmentación parlamentaria ha dejado más espacio a la Asamblea y son más necesarios los acuerdos para gobernar, pero no lo es menos que la figura del Presidente del Gobierno sigue estando por encima de cualquier otra y que nuestra forma de gobierno podría caracterizarse como un "parlamentarismo presidencialista" (Aragón Reyes, 2002: 49). La consecuencia de ello es la progresiva reducción del Parlamento "a la condición de máquina de legislar dominada por el Gobierno y el traslado de la hegemonía al gabinete primero y al líder de la mayoría después" (Martínez Sospedra, 1978: 206).

La doctrina ha escrito que actualmente el conflicto político no se puede entender en términos interorgánicos (Sánchez Navarro, 1995: 224). Más que hablar de la relación Gobierno-Parlamento habría que hacerlo de la relación Gobierno- 
oposición o mayoría-minoría. Debido a ello se debería diferenciar entre control "en" y "por" el Parlamento. Dado que muchos de los instrumentos de fiscalización en sentido estricto dependen del principio de mayoría, se ha de entender que el control se ejerce en toda la actividad del Parlamento, esté destinada o no al control como tal. Por eso se puede distinguir entre Parlamento entendido como órgano, destinado a la formación de la voluntad popular, y Parlamento como institución, que es donde se debate la acción del Gobierno (Rubio Llorente, 2012: 687 y ss.). Aunque también hay autores que piensan que "el control por naturaleza es responsabilidad de la mayoría parlamentaria, por cuanto en un sistema democrático y por medio de una votación, sólo la mayoría es capaz de alcanzar una propia decisión" (Embid Irujo, 1992: 11).

Pero la realidad es que actualmente existe la sensación de que "el control es ineficaz, porque la mayoría que apoya al Gobierno no fiscaliza su actuación" (Fernández Sarasola, 2000: 90). Y es que las iniciativas de control que realiza el Grupo o Grupos Parlamentarios de apoyo al Gobierno, en la mayoría de ocasiones, poco aportan a la función de control o, lo que sería peor, "ayudan a hacer oposición de la oposición" (Aranda Álvarez, 2007: 109). Esto es así porque no existe una disponibilidad real de los instrumentos de control para las minorías y se apunta que es necesario una adaptación a las nuevas realidades sociales de estas instituciones para consolidar las funciones que cumplen (López Guerra, 2002: 32).

Hoy la principal función de los Parlamentos es la de controlar la dirección política del Gobierno. Tal fiscalización es ejercida principalmente por las minorías parlamentarias, por lo que se puede decir que el control y el Parlamento son "el hábitat natural de la oposición y las minorías, y un contrapeso imprescindible del eje mayoría-Gobierno" (García Roca, 2017: 12). Pero la situación generada por la covid-19 ha supuesto un problema a la hora de controlar al Gobierno, de lo que se habla a continuación.

III. REACCIÓN DE LOS PARLAMENTOS ANTE LA COVID-19. ESPECIAL REFERENCIA AL CONGRESO DE LOS DIPUTADOS

Las soluciones adoptadas por los Parlamentos autonómicos y por las Cortes Generales para hacer frente a la situación generada por la covid-19 han sido variadas. En los primeros compases del estado de alarma la totalidad de 
fundación

Manuel Giménez Abad

deEstudios ParlamentariosydelEstado Autonómico

Asambleas Legislativas decidieron prácticamente su cierre, al entender que también se veían afectadas por las medidas sanitarias adoptadas en los peores momentos de la pandemia, por lo que se empezó a mirar qué posibilidades ofrecía la tecnología para suplir tales carencias (Tudela Aranda, 2020: 7). Pero luego se han ido tomando otras medidas para retomar su funcionamiento, que merece la pena indicar ahora.

Algunos Parlamentos autonómicos prevén en sus reglamentos la posibilidad de delegar el voto. Esta ha sido una solución que se ha planteado en los Parlamentos del País Vasco, Navarra o Cataluña e, incluso, en otros lugares, como el Parlamento de Andalucía, se ha reformado su reglamento una vez se pudo reunir de nuevo el Pleno para introducir esta posibilidad "en tanto existan medidas de confinamiento y/o distancia de seguridad entre personas, consecuencia de pandemias u otras situaciones de excepcional gravedad" (artículo 90 bis). Dicha institución -la delegación de voto- es, cuanto menos, discutible. Parte de la doctrina ha defendido que sí que se puede introducir en el ámbito parlamentario autonómico, dado que la personalidad e indelegabilidad del voto está únicamente prevista para diputados y senadores (artículo 79.3 CE), además de que no existe un mandato constitucional por el cual se obligue a que la organización y funcionamiento de los Parlamentos autonómicos sea idéntica a la de las Cortes Generales (STC 179/1989, FJ 6). Y además, con esta posibilidad se soluciona que, cuando concurran circunstancias que puedan dificultar el ejercicio del cargo representativo, se garantice el derecho de los ciudadanos a intervenir políticamente (Presno Linera y Ortega Santiago, 2009: 44-47). Pero también cabría argumentar que dicho precepto se ha de aplicar a los parlamentarios autonómicos, dado que cumplen con funciones análogas a las de diputados y senadores, esto es, legislativas, al igual que se les aplica la prohibición de mandato imperativo prevista en el artículo 67.2 CE, algo que sería más cuestionable en el ámbito local (Dueñas Castrillo, 2016: 148); y la personalidad e indelegabilidad del voto, aunque sólo estén reconocidas constitucionalmente para diputados y senadores, no implica que "no tengan aplicación al modelo de democracia representativa que caracteriza a las instituciones parlamentarias autonómicas" (De Miguel Bárcena, 2010: 150).

Las anteriores críticas se pueden extender a otras soluciones ofrecidas por algunos Parlamentos autonómicos, como el voto ponderado, adoptado en la Asamblea de Murcia. En este caso se ha argumentado que con dicha solución "no se produciría en ningún caso una alteración en los resultados de las votaciones y 
fundación

Manuel Giménez Abad

deEstudios ParlamentariosydelEstado Autonómico

que estos siempre serían adecuados a la composición de la Cámara y a las mayorías constituidas en cada ocasión" (Fernández de Simón Bermejo, 2020: 152), pero es lo mismo que decir que el diputado individual no tiene ninguna operatividad y que los portavoces de cada Grupo Parlamentario podrían votar con un determinado porcentaje de votos, que sería el mismo que el de escaños que tuviera su Grupo. Algo así podría vulnerar el ius in officium de los diputados y, por tanto, el artículo $23 \mathrm{CE}$.

Existen medidas menos lesivas para garantizar el derecho del artículo 23 CE que también se han adoptado en los Parlamentos autonómicos. Por ejemplo, el voto no presencial o voto telemático, que se ha ampliado para el supuesto de imposibilidad de acudir al Parlamento debido a la situación de pandemia en las Cortes de Aragón y en las de Castilla y León; o la celebración telemática de las sesiones de las Comisiones con votaciones efectuadas por llamamiento, como ha sucedido en las Cortes de Aragón, lo cual, para autores como Rubio del Val y Blasco Jáuregui, sería conforme a la jurisprudencia constitucional en casos como el presente (Rubio del Val y Blasco Jáuregui, 2020: 48). Esta previsión -la de la celebración por videoconferencia u otros sistemas técnicos de las sesiones de las Comisiones- ya estaba presente en otros reglamentos como el del Parlamento de las Islas Baleares, si bien en este caso es debido a que la meteorología en muchas ocasiones impide a los diputados de algunas islas desplazarse hasta la sede del Legislativo (Ridao Martín, 2020: 76). Pero estas soluciones tecnológicas tienen problemas importantes, como ocurrió en la sesión plenaria de 23 de abril de la Asamblea de Madrid, que tuvo que ser suspendida "antes de que transcurriera una hora desde que fuera abierta, por los cortes producidos durante la conexión, que impidieron su correcto desarrollo" (Greciet García, 2020: 76).

En muchos casos se ha acudido a la Diputación Permanente como forma de mantener la actividad parlamentaria. El ordenamiento constitucional español optó por esta institución para prever una solución en los momentos de inactividad parlamentaria, dado que "el sistema político español (...) lleva implícito un sistema de colaboración entre el Parlamento y el Gobierno, pero, para que ello sea posible, es necesario que ambos funcionen" (Alós Martín, 1989: 39). La principal función de la Diputación Permanente es velar por los poderes de la Cámara, que es "una función de defensa de la integridad constitucional y de aquellas situaciones que supongan un peligro para las potestades del Parlamento" (Mellado Prado, 1988: 71). Es decir, esta es una forma de organización y funcionamiento de las Cámaras, pero únicamente está prevista para dos 
fundación

Manuel Giménez Abad

deEstudios Parlamentariosydel Estado Autonómico

supuestos: cuando el Parlamento se encuentre fuera de los períodos ordinarios de sesiones o cuando haya finalizado su mandato (Seseña Santos, 2016: 224). Por esa misma razón sus atribuciones están limitadas: la Diputación Permanente en ningún caso sustituye a la Cámara. El caso de que el Parlamento no pueda reunirse debido a una pandemia como la actual no está previsto como supuesto para que pueda actuar la Diputación Permanente. Sin embargo, podría regularse en los reglamentos parlamentarios tal situación, si bien es cierto que su duración debería ser mínima, pero con ello se podría garantizar una presencia de la institución parlamentaria "en aquellas situaciones cuyo abandono, aunque sea temporal, pone en manos del Ejecutivo un poder excesivo y aquellas otras sin cuyo ejercicio se perturbaría la funcionalidad del sistema en su conjunto" (Mellado Prado, 1988: 49). Además, el Parlamento, en atención a su autonomía, puede organizar sus trabajos de tal manera que suspenda el período de sesiones en el que se encontraba cuando se declare una pandemia que impida a los ciudadanos desplazarse libremente. Pero ello es sumamente peligroso si se tiene en cuenta que cualquiera de los estados regulados en el artículo $116 \mathrm{CE}$, que son los únicos capaces de limitar o suspender derechos fundamentales, puede otorgar al Ejecutivo un poder mucho más grande del que tiene en situaciones ordinarias y que, entonces, es aún más importante, si cabe, la labor de fiscalización que ejerce el Parlamento.

Volviendo al plano estatal, hay que tener en cuenta que no es la primera vez que se plantean problemas de desplazamiento de los diputados y soluciones para solventarlos. El artículo 109 de la Constitución de 1812 disponía que "si la guerra o la ocupación de alguna parte del territorio de la Monarquía por el enemigo impidieren que se presenten a tiempo todos o algunos de los Diputados de una 0 más provincias, serán suplidos los que falten por los anteriores Diputados de las respectivas provincias, sorteando entre sí hasta completar el número que les corresponda".

En el Congreso de los Diputados, la Mesa suspendió las sesiones que estaban previstas para la semana del 10 de marzo y la actividad parlamentaria se redujo a mínimos durante un mes, una vez concluida la primera prórroga del estado de alarma el 12 de abril. Durante ese tiempo también se suspendieron los plazos de las iniciativas que se encontraban en tramitación. Sólo fue en el momento en el que el Congreso autorizó una nueva prórroga, el 9 de abril, cuando tal suspensión de la actividad se hizo más insostenible y se levantaron algunas de las restricciones impuestas. Mientras tanto, el Congreso también adoptó alguna de 
las soluciones ofrecidas por las Asambleas Legislativas autonómicas. De esta forma, la Mesa acordó el 19 de marzo que todos los diputados que lo desearan podían emitir su voto por el procedimiento telemático en las sesiones plenarias de ese mes (esto también sucedió en el Senado, donde la Mesa extendió dicha posibilidad el 12 de marzo en relación con la autorización de un convenio internacional incluido en el orden del día de la siguiente sesión plenaria que se tenía que celebrar). Lo que sí que se rechazó desde un primer momento fue la posibilidad de intervenir por videoconferencia en las sesiones plenarias, como informa una nota de la Secretaría General del Congreso de 25 de marzo. Hay que tener en cuenta que las SSTC 19 y 45/2019 "han subrayado la inmediatez y presencialidad de la actividad parlamentaria, aun admitiendo la posibilidad de que los Reglamentos prevean el voto en ausencia cuando concurran circunstancias excepcionales o de fuerza mayor" (García-Escudero Márquez, 2020: 19). Fue finalmente el 7 de abril, día en el que la Mesa del Congreso acuerda el levantamiento de la suspensión del cómputo de plazos en la tramitación de iniciativas, con efectos desde el día 13 de ese mes, cuando se reactiva la labor parlamentaria y, con ello, la Junta de Portavoces acuerda reanudar las sesiones de control al Gobierno. Por otro lado, tanto Mesa como Junta de Portavoces, se han venido reuniendo de manera telemática, algo que no plantea problemas, dado que "organizan el trabajo pero no ejercen las funciones constitucionales 0 estatutarias (...), a diferencia de Pleno, comisiones y Diputación Permanente" (García-Escudero Márquez, 2020: 25) ${ }^{1}$.

Lo cierto es que la falta de previsión de los Reglamentos de las Cámaras para adecuar su funcionamiento a situaciones como la vivida ha hecho que hayan cobrado "especial importancia los acuerdos de las Mesas de las Cámaras, como instrumentos que han dotado de especial flexibilidad a las normas de organización y funcionamiento internas, permitiendo con ello la adaptación de las Cortes Generales a las nuevas circunstancias" (García de Enterría Ramos y Navarro Mejía, 2020: 259).

Si se tiene en cuenta lo anterior, no cabe duda de que el normal funcionamiento de las Cortes Generales se vio alterado como consecuencia de la declaración del estado de alarma por covid-19. Algo que, como se verá en el siguiente epígrafe, ha tenido consecuencias en las relaciones entre Ejecutivo y Legislativo. Pero es

\footnotetext{
${ }^{1}$ Para el relato de lo que ha ocurrido en el Congreso y Senado en este apartado he seguido a García Escudero-Márquez, P., "Actividad y funcionamiento de las Cortes Generales durante el estado de alarma por COVID-19”, Cuadernos Manuel Giménez Abad, monografía núm. 8, 2020.
} 
que además esta circunstancia podría haber contradicho lo dispuesto en el apartado 5 del artículo $116 \mathrm{CE}$ in fine, esto es, que el funcionamiento del Parlamento estatal, junto con los demás poderes constitucionales del Estado, "no podrán interrumpirse durante la vigencia de estos estados"; o lo establecido en el artículo 1.4 de la Ley Orgánica 4/1981 reguladora de los estados de alarma, excepción y sitio, que determina que "la declaración de los estados de alarma, excepción y sitio no interrumpe el normal funcionamiento de los poderes constitucionales del Estado".

\section{LA FISCALIZACIÓN AL GOBIERNO DURANTE EL ESTADO DE ALARMA POR COVID-19}

Se pueden distinguir dos momentos en la función de control al Gobierno durante el estado de alarma por covid-19. El primero, cuando aún estaban vigentes los acuerdos de las Mesas de Congreso y Senado que suspendieron las sesiones plenarias y de comisiones, en el que tan sólo se celebraron los Plenos en el Congreso de prórroga del estado de alarma y de convalidación de decretos-leyes. Y un segundo momento, a partir del levantamiento por las Mesas de la suspensión del cómputo de plazos con efectos desde los días 13 de abril -en el Congreso- y 14 de abril -en el Senado-, que conllevó que a partir de entonces se añadieran al orden del día de los Plenos las preguntas e interpelaciones a los miembros del Gobierno.

En el que se acaba de denominar primer momento se realizaron tres Plenos en el Congreso de los Diputados con las medidas de seguridad oportunas (limitaciones en la asistencia a aquellos diputados que debían intervenir y voto telemático para los restantes, en el caso de que fuera necesario), y tan sólo para ventilar temas relacionados con el estado de alarma por covid-19. El primero de ellos tuvo lugar el 18 de marzo de 2020 con un único punto en el orden del día: "comunicación del Gobierno que acompaña al Real Decreto 463/2020, de 14 de marzo, por el que se declara el estado de alarma para la gestión de la situación de crisis sanitaria ocasionada por el covid-19"2. El segundo, celebrado el 25 de marzo, debatió, además de la primera prórroga del estado de alarma, la convalidación de los primeros reales decretos-leyes para hacer frente a la situación de pandemia ${ }^{3}$. Y el

\footnotetext{
${ }^{2}$ http://www.congreso.es/backoffice_doc/atp/orden_dia/pleno_014_18032020.pdf

${ }^{3} \mathrm{http}: / /$ www.congreso.es/backoffice_doc/atp/orden_dia/pleno_015_25032020.pdf
} 
fundación

Manuel Giménez Abad

deEstudios ParlamentariosydelEstado Autonómico

tercero, celebrado el 9 de abril, en el que se autorizó la segunda prórroga del estado de alarma y se discutió la convalidación de otros tres reales decretosleyes ${ }^{4}$.

Si observamos los órdenes del día de los Plenos, se puede decir que únicamente se han tratado aquellas cuestiones que eran constitucionalmente obligadas. En cuanto al estado de alarma, no cabe duda de que, conforme al artículo $116 \mathrm{CE}$, "si hay una Cámara que debe ser protagonista durante el estado de alarma es el Congreso de los Diputados" (García-Escudero Márquez, 2020: 20). Aunque se hubiera decidido que el funcionamiento normal de la Cámara Baja quedaba suspendido debido a la pandemia, el control al Gobierno realizado a través de la autorización de las dos primeras prórrogas del estado de alarma, además de obligatorio, era sumamente importante si se tiene en cuenta que el Ejecutivo contaba con un poder mucho mayor que el habitual, sobre todo en un Estado descentralizado territorialmente como el nuestro. La única otra forma de control -en sentido estricto- al Gobierno y de rendición de cuentas que se realizó durante este primer mes de estado de alarma fueron las comparecencias semanales del Ministro de Sanidad ante su Comisión correspondiente (García de Enterría Ramos y Navarro Mejía, 2020: 264) y las preguntas con respuesta escrita, que se han podido seguir registrando y contestando en cualquier momento, aunque el Gobierno no tenía obligación de hacerlo en los 30 días previstos en los reglamentos de Congreso y Senado porque los plazos estaban suspendidos (García de Enterría Ramos y Navarro Mejía, 2020: 281).

La otra manera de control en el Parlamento durante esta primera fase del estado de alarma ha sido a través de la convalidación de los decretos-leyes, tal y como establece nuestra Carta Magna. Aunque se hable de ello en otra parte del libro, cabe apuntar ahora que la doctrina ha indicado que, debido a la relajación del presupuesto habilitante del decreto-ley, este instrumento ha pasado a ser un cauce normativo alternativo al procedimiento legislativo ordinario en vez de una figura excepcional (García Majado, 2017: 236). Y es que es cierto que no se puede discutir la virtualidad y necesidad de que el Gobierno haga uso del decretoley, entendiéndose como una excepción al principio de separación de poderes (Enériz Olaechea, 2011: 5). Sin embargo, lo que estaba previsto como un supuesto excepcional "se ha deslizado hacia una cierta anormal normalidad, asumida con mucha frecuencia por el TC" (Martín Rebollo, 2015: 24), hasta el punto de que "la ocupación normal por el decreto-ley del espacio de la ley es hoy

\footnotetext{
${ }^{4}$ http://www.congreso.es/backoffice_doc/atp/orden_dia/pleno_016_09042020.pdf
} 
una realidad innegable" (Aragón Reyes, 2016: 14). Lo precedente, además de la excesiva laxitud de la interpretación del presupuesto habilitante por parte del Tribunal Constitucional, como se ha apuntado ligeramente, se debe a la debilidad de nuestro sistema parlamentario (Aragón Reyes, 2016: 14). Esto podría haberse visto agravado en la situación del estado de alarma por covid-19, aunque aquí los decretos-leyes estarían más justificados si se tiene en cuenta la situación de emergencia del momento, pero no es menos cierto que quizás se haya hecho un uso abusivo de esta manera de legislar durante dicho período.

A partir del momento en el que se levantó por la Mesa del Congreso la suspensión del cómputo de plazos con efecto desde el 13 de abril de 2020, como se ha dicho, se volvieron a añadir las preguntas e interpelaciones a los miembros del Gobierno a través de los denominados Plenos de control, aunque "se optó por no incluir mociones consecuencia de interpelaciones ni proposiciones no de ley, como consecuencia de las dificultades que planteaba el voto de estas cuestiones de manera telemática" (García de Enterría Ramos y Navarro Mejía, 2020: 263). Desde entonces se han celebrado un total de 12 sesiones plenarias, de las cuales 9 han contenido preguntas e interpelaciones a quienes integran el Ejecutivo, a lo que hay que añadir que diferentes Ministros comenzaron a acudir de manera habitual a sus respectivas Comisiones para informar de las actuaciones llevadas a cabo en la gestión de la crisis en sus diferentes ramas ministeriales. El Pleno del 16 de junio, estando aún vigente el estado de alarma, pero con unas medidas mucho más moderadas que con anterioridad, fue la primera sesión que reanudó el debate de proposiciones no de ley y de mociones consecuencia de interpelaciones urgentes ${ }^{5}$.

El caso del Senado ha sido diferente, sobre todo debido a que no tiene ningún protagonismo en relación con el estado de alarma en comparación con el Congreso. El 12 de marzo la Mesa decidió no convocar sesiones plenarias ni de comisiones mientras se encontrara vigente el estado de alarma. Pero, al igual que sucedió en la Cámara Baja, con el levantamiento de la suspensión del cómputo de plazos acordada por la Mesa el 14 de abril, también se autorizó la convocatoria de la primera sesión plenaria desde que se decretara el estado de alarma (a excepción de la celebrada el 17 de marzo, que sólo tuvo como objeto la autorización del Protocolo al Tratado del Atlántico Norte sobre la adhesión de la

\footnotetext{
${ }^{5}$ http://www.congreso.es/backoffice_doc/atp/orden_dia/pleno_028_16062020.pdf
} 
República de Macedonia del Norte ${ }^{6}$ ). Con la celebración del Pleno del 21 de abril, vuelve el control de la Cámara Alta al Gobierno. Junto con el de este día, se han celebrado un total de 5 Plenos, en los que se han incluido preguntas e interpelaciones a los miembros del Gobierno. Ha sido en la sesión celebrada el día 16 de junio, al igual que ha ocurrido en el Congreso, cuando se han empezado a debatir otras formas de control, en este caso una moción derivada de una interpelación ${ }^{7}$.

Si se tiene en cuenta todo lo dicho hasta ahora, se puede afirmar que la capacidad de las Cortes Generales para controlar al Gobierno se ha visto mermada y, en el momento más crítico de la pandemia, únicamente se ha reducido a los actos de autorización de la prórroga del estado de alarma y el debate de convalidación de los reales decretos-leyes. Para algunos autores, a pesar de que el funcionamiento normal de las Cortes Generales se haya visto afectado, durante todo este periodo no se ha dejado de realizar la labor de control, sino que se ha tratado de "adecuar esta función de control a las exigencias fácticas del estado de alarma" (García de Enterría Ramos y Navarro Mejía, 2020: 280). Pero para otros, las diferentes medidas adoptadas ha provocado en muchos casos que "en un momento de máxima concentración del poder, cuando el control era más necesario que nunca, el Parlamento casi desaparecía de la escena" (Tudela Aranda, 2020: 7). Dicho de otra manera, se ha producido "la paradoja de que, contra un mayor poder ejecutivo, hubo una especie de vacío parlamentario, al menos en un primer momento, pues desaparece, en parte, la función de orientación y control" (Fernández Gutiérrez, 2020: 64).

\section{CONCLUSIONES}

Actualmente, la función de control al Gobierno es la más importante de las que ejerce el Parlamento y, más en concreto, la oposición. Pero se ha visto estrechada durante el estado de alarma por covid-19.

Los Parlamentos españoles, tanto los autonómicos como las Cortes Generales, han reaccionado de diversas maneras a la situación generada por la pandemia. De manera general, en las primeras semanas su funcionamiento fue mínimo y

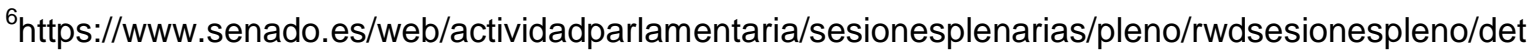
alle/index.html?id=6\&legis $=14$

${ }^{7} \mathrm{https}: / /$ www.senado.es/web/actividadparlamentaria/sesionesplenarias/pleno/rwdsesionespleno/det alle/index.html?id=12\&legis $=14$
} 
apenas tuvieron ninguna actividad. Más adelante se han tratado de buscar diversas soluciones, donde los órganos de gobierno de las Cámaras han jugado un papel muy relevante, como la delegación de voto, el voto telemático, la celebración de sesiones telemáticas o la activación de la Diputación Permanente. Pero las medidas llevadas a cabo han reducido las capacidades de las Asambleas Legislativas y, en algunos casos, han impedido que desarrollasen sus funciones con una cierta normalidad, a pesar de la pandemia.

En el ámbito estatal, ha habido dos momentos diferenciados en lo que a la fiscalización del Ejecutivo se refiere. El primero, en el cual apenas hubo control por parte del Parlamento al Gobierno más allá de los mecanismos constitucionalmente establecidos para la autorización de las prórrogas del estado de alarma y la convalidación de decretos-leyes, hasta que se decidió el levantamiento de la suspensión de los plazos parlamentarios por las Mesas y la reanudación de los llamados Plenos de control. A partir de entonces, aproximadamente desde que se aprobara la segunda prórroga del estado de alarma, comienza el segundo momento, en el que el control se ha llevado a cabo con mayor normalidad, aunque no se haya tramitado ninguna iniciativa de impulso o indirizzo político al Gobierno hasta casi el fin del estado de alarma, debido a las dificultades de los parlamentarios para ejercer el voto.

Como conclusión general se puede afirmar el normal funcionamiento de las Cortes Generales y las relaciones entre Gobierno y Parlamento se han visto alteradas durante el estado de alarma por covid-19. Hasta la fecha no se había vivido en la democracia española de 1978 una situación en la cual el Ejecutivo contara con un poder tan grande, pero sin embargo el Legislativo no ha podido realizar su función de control con las suficientes garantías. Es cierto que tanto Congreso y Senado han debido adaptar su normal funcionamiento al contexto generado por la pandemia, pero no lo es menos que sería deseable que se reformasen los reglamentos parlamentarios para contemplar soluciones más adecuadas a momentos como el vivido entre marzo y junio de 2020. De esta forma se podría evitar que el Gobierno pudiese llevar a cabo medidas políticas sin los correspondientes frenos y contrapesos con los que ha de contar cualquier Estado democrático de Derecho. 
fundación

Manuel Giménez Abad

deEstudios Parlamentarios ydelEstado Autonómico

VI. BIBLIOGRAFÍA

- ALÓS MARTíN, I., "La Diputación Permanente en la Constitución española", Revista de las Cortes Generales, núm. 18, 1989.

- ARAGÓN REYES, M., “¿Un parlamentarismo presidencialista?”, Claves de Razón Práctica, núm. 123, 2002.

- ARAGÓN REYES, M., "Uso y abuso del Decreto-Ley: una propuesta de reinterpretación constitucional", en El Cronista, núm. 60, 2016.

- ARANDA ÁLVAREZ, E., "Transformaciones en los instrumentos de control parlamentario", Teoría y Realidad Constitucional, núm. 19, 2007.

— DE MIGUEL BÁRCENA, J., "La personalidad e indelegabilidad del voto y las reformas de los reglamentos de los Parlamentos autonómicos", en Revista Española de Derecho Constitucional, núm. 90, 2010.

- DUEÑAS CASTRILLO, A. I., "La incidencia de los partidos políticos en el revocatorio del mandato en los Ayuntamientos españoles", BIGLINO CAMPOS, P. (coord.), Partidos políticos y mediaciones de la democracia directa, Centro de Estudios Políticos y Constitucionales, Madrid, 2016

- EMBID IRUJO, A., "El control parlamentario del Gobierno y el principio de la mayoría parlamentaria. Algunas reflexiones", Revista de las Cortes Generales, núm. 25, 1992.

— ENÉRIZ OLAECHEA, F. J., "EI Decreto-Ley autonómico: regulación y crítica", en Revista Aranzadi Doctrinal, ㄲo 10, 2011.

- FERNÁNDEZ DE SIMÓN BERMEJO, E., "La actuación de la Asamblea Regional de Murcia desde la declaración del estado de alarma por la crisis sanitaria provocada por el COVID-19", Cuadernos Manuel Giménez Abad, monografía núm. 8, 2020

- FERNÁNDEZ GUTIÉRREZ, M., "La Junta General del Principado de Asturias bajo la pandemia ocasionada por la COVID-19", Cuadernos Manuel Giménez Abad, monografía núm. 8, 2020.

- FERNÁNDEZ SARASOLA, I., "El control parlamentario y su regulación en el ordenamiento español", Revista Española de Derecho Constitucional, núm. 60, 2000 . 
- GARCÍA DE ENTERRÍA RAMOS, A., y NAVARRO MEJÍA, I., "La actuación de las Cortes Generales durante el estado de alarma para la gestión de la crisis del COVID-19", Revista de las Cortes Generales, núm. 108, 2020.

- GARCÍA ESCUDERO-MÁRQUEZ, P., "Actividad y funcionamiento de las Cortes Generales durante el estado de alarma por COVID-19", Cuadernos Manuel Giménez Abad, monografía núm. 8, 2020.

- GARCÍA MAJADO, P., "Del uso al abuso: el decreto-ley en la España democrática", Diálogos Jurídicos: Anuario de la Facultad de Derecho de la Universidad de Oviedo, núm. 2, 2017.

- GARCÍA ROCA, F. J., "Control parlamentario y convergencia entre presidencialismo y parlamentarismo", Revista Mexicana de Derecho Constitucional, núm. 37, 2017.

- GRECIET GARCÍA, E., "La Asamblea de Madrid y el COVID-19: lo que nunca debió suceder, Cuadernos Manuel Giménez Abad, monografía núm. 8, 2020

- LÓPEZ GUERRA, L., "Organización y funcionamiento del Parlamento del futuro", Pau i Vall, F. (coord.), El Parlamento del Siglo XXI. VIII Jornadas de la Asociación Española de Letrados de Parlamentos, Tecnos, Valencia, 2002.

- MARTíN REBOLLO, L., "Uso y abuso del Decreto-Ley. (Un análisis empírico)", en Revista Española de Derecho Administrativo, núm. 174, 2015.

- MARTínEZ SOSPEDRA, M., "El régimen parlamentario y el sistema de partidos", Ramírez, M. (ed.), El control parlamentario del Gobierno en las democracias pluralistas, Labor, Madrid, 1978.

- MELlado PRADO, P., "El problema de la continuidad del Parlamento: la Diputación Permanente", Revista de Derecho Político, núm. 27-28, 1988.

- PRESNO LINERA, M. y Ortega Santiago, C., La sustitución temporal de los representantes políticos, Centro de Estudios Políticos y Constitucionales, Madrid, 2009.

- REQUEJO RODRíGUEZ, P., "La posición de las minorías en el Estado democrático", Gutiérrez Gutiérrez, I. (ed.), Decidir por mayoría, Marcial Pons, Madrid, 2016.

- RIDAO MARTÍN, J., "Virtualizando el Parlamento (hasta donde se puede). El régimen del Parlamento de Cataluña durante la crisis de la COVID-19 y las reformas tecnológicas y reglamentarias operadas para regular la actividad no 
presencial en el futuro", Cuadernos Manuel Giménez Abad, monografía núm. 8, 2020.

- RUBIO DEL VAL, C. y BLASCO JÁUREGUI, J., “El papel del Parlamento autonómico en el estado de alarma: crónica de la actividad de las Cortes de Aragón", Cuadernos Manuel Giménez Abad, monografía núm. 8, 2020.

- RUBIO LLORENTE, F., La forma del poder. Estudios sobre la Constitución, Centro de Estudios Políticos y Constitucionales, 3aㅗ ed., Madrid, 2012.

- SÁNCHEZ NAVARRO, A. J., "Control parlamentario y minorías", Revista de Estudios Políticos (Nueva Época), núm. 88, 1995.

- SESEÑA SANTOS, L., "El Pleno y la Diputación Permanente", Sarmiento Méndez, X. A. (coord.), Manual AELPA del Parlamentario, AELPA-Wolters Kluwer, Madrid, 2016.

- TUDELA ARANDA, J., "Parlamento y crisis sanitaria", Cuadernos Manuel Giménez Abad, monografía núm. 8, 2020. 